RAD Conference Proceedings, vol. 2, pp. 159-162, 2017

www.rad-proceedings.org

novororitty

\title{
BEHAVIOR OF MODERN INTEGRATED CIRCUITS AFTER LATCH-UP PARRYING
}

\author{
P.K. Skorobogatov ${ }^{1,2}$, G.G. Davydov ${ }^{1,2}{ }^{*}$, A.A. Pechenkin',2, D.V. Boychenko² \\ ${ }^{1}$ Institute of Extreme Applied Electronics of National Research Nuclear University "MEPhI”, Moscow, Russia \\ ${ }^{2}$ JSC “Specialized Electronic Systems”, Moscow, Russia
}

\begin{abstract}
The work is devoted to the research of the single and multiple latch-up impact on the subsequent behavior of electrical parameters and operability of modern integrated circuits. The impact of interrupted SEL amount on subsequent behavior of IC is introduced. An approach to the estimation of the maximum time in the latch-up state is formulated to ensure IC's fault-free operation after SEL.
\end{abstract}

Key words: Latch-up, integrated circuit, failure, damage

DOI: $10.21175 /$ RadProc.2017.32

\section{INTRODUCTION}

The single event latch-up (SEL) in integrated circuits (IC) is a well-known effect. Nowadays, physical and analytical models of SEL manifestation, as well as methods for SEL mitigation, based on both layout and schematic approaches, are developed [1-3]. However, the impact of time in latch-up state, as well as the number of interrupted SELs, on subsequent behavior of IC's parameters and functionality, is still not clear.

It is well known that even single latch-up may cause an irreversible failure of destructive changes in IC's behavior. In few works [4,5], the so-called latent damage as a result of the SEL is discussed, and the experimental data of IC reliability decrease after SEL is presented. Moreover, in [4], an increase in the possibility of the occurrence of such effects with a decrease of process size is mentioned.

To estimate the possibility of IC failure after SEL, one can use the survival testing procedure [6]. During the survival testing, the devise-under-test (DUT) is exposed by series of SELs with increasing the time in latch-up state (a number of values from a few milliseconds to tens of minutes). As a result of survival testing, the coarse estimation of maximum time in SEL state without the subsequent failure or damage may be obtained. Such information is needed to form requirements for the power supply and facilities of SEL mitigation.

The survival testing for more than 1000 types of electronic devices was performed in IEAE NRNU $\mathrm{MEPhI}$ in the period from 2009 to 2016. For about 100 of them the irreversible failure was found after single or multiple SEL. Few results are in table 1.
The analysis of the results shown above allows one to mark the following:

1. Irreversible failure and/or damage is usually found in the range from tens of $\mu$ s to tens of seconds;

2. There is no clear correlation between time in latch-up state and IC functional and fabrication process;

3. Necessary SEL parrying time may be few microseconds in some cases; this value is less than can be provided by modern latch-up current limiting ICs (such as 3DPMo168-2-xx) [6]. Moreover, there is not assurance that the current limiting ICs themselves have internal latch-up immunity.

Table 1. Time in SEL state before failure

\begin{tabular}{|l|c|}
\hline \multicolumn{1}{|c|}{ IC type } & Time in SEL state before failure, $\mathrm{s}$ \\
\hline XC95144-15TQ100I & 0.000003 \\
\hline 74AC244SC & $<0.1$ \\
\hline XQ4010E-4CB196M & 1 \\
\hline ADT7518ARQZ & 10 \\
\hline TPS70158PWP & 20 \\
\hline XCV50-4TQ144I & 40 \\
\hline SN65HVD233HD & 70 \\
\hline AM29F160DT-75EF & 120 \\
\hline
\end{tabular}

The analysis of whole results allows also the following conclusions:

4. SEL manifestation may be followed by several statistically-independent and irreversible effects:

- increasing of the supply current;

- operation failures;

- latch-up disappear after first SEL.

5. In few cases, in accordance with [4,5], one can see the failure after series of SEL.

*ggdav@spels.ru 
Thus, despite the considerable amount of experimental data, there is still no solution to the following problems:

- estimation of the IC failure probability after single or series latch-up in dependence of time and maximum current in SEL-state;

- estimation of maximum time in SEL-state to guarantee the failure-free subsequent IC functioning.

In this work, an approach to solve these problems is discussed.

\section{RESEARCH METHODS AND FACILITIES}

The comprehensive study of SEL requires a possibility of setting its parameters (time in latch-up state and maximum SEL current) precisely. A specialized testing system was developed to meet these requirements. The system includes the laser source with a positioning subsystem [6] as the SEL initiator, as well as a programmable SEL-parrying module (see Fig. 1). The system was developed on the basis of PXIplatform (National Instruments, USA) [8]: power supply PXI-4110 with auxiliary power source, digital multimeter PXI-4071, scope PXI-5114, FPGA-based acquisition board PXI-7851R.

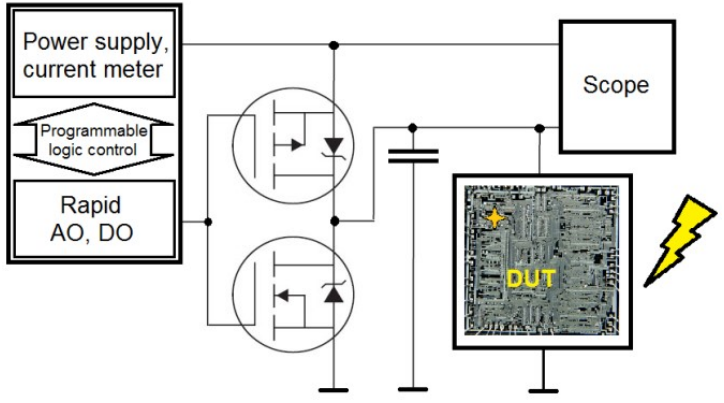

Figure 1. Simplified scheme of the testing system

System features:

- variable time in latch-up state (from a few microseconds to 20 minutes), time set precise is $1 \mu \mathrm{s}$;

- measurements of IC's supply current (also in SEL-state) with $1 \mathrm{MHz}$ frequency;

- possibility of voltage-current SEL characteristics rapid measurement.

After each SEL detection, the countdown of the time in SEL state is started. After this time expired, the supply voltage turns to oV, and after OFF time has expired, turns to active value again. For accurate study, the same time in SEL state must be set for at least 2 samples. The estimation of prognosis error requires much more samples to be studied.

\section{Device Under Test}

In this work, the CMOS IC of the digital thermometer with 4-ch $\mathrm{ADC}$ and $\mathrm{I} 2 \mathrm{C} \mathrm{AD} 7518$ was chosen. For this IC time of survivability after SEL was previously obtained and is about 10 seconds. Such the time allows researcher to set time of SEL parrying more accurately.
Some investigations were performed with $\mathrm{AD} 7518$ earlier. SEL-sensitive areas were found by using the method described in [7] (Fig. 2). Search of SELsensitive regions was performed on separate group of samples with relatively long SEL-parrying time.

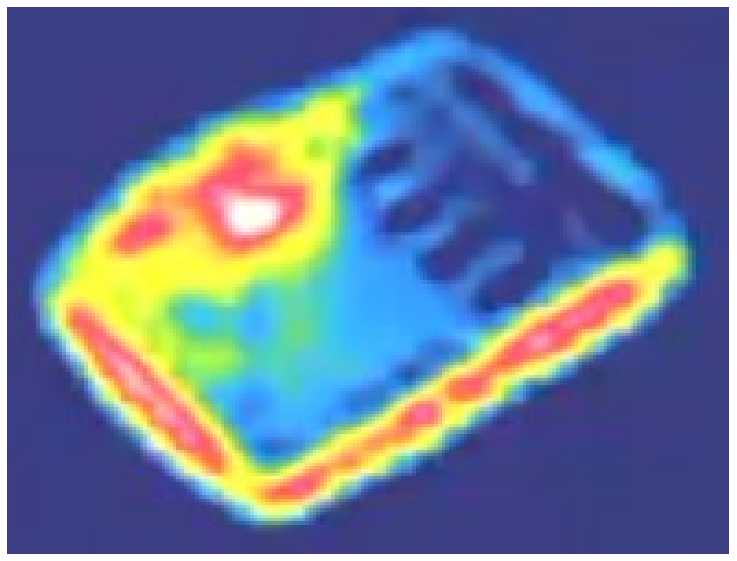

(a)

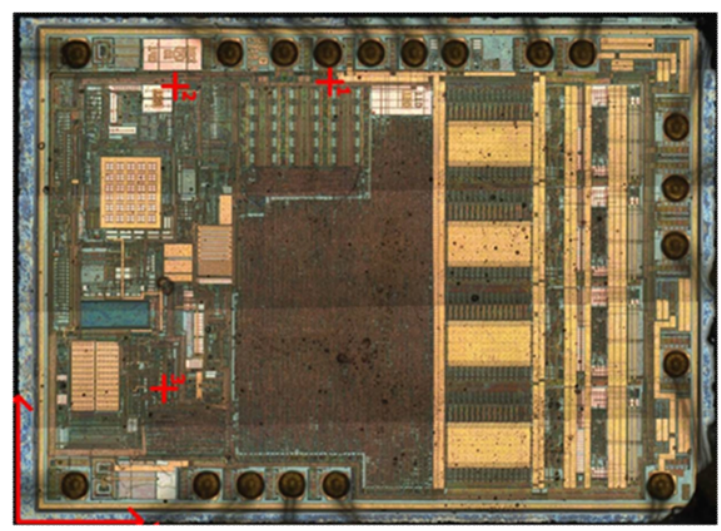

(b)

Figure 2. Thermal image of SEL, 0.2s after SEL initiate (a), and SEL-sensitive areas in ADT7518 shown on chip photo (b)

\section{EXPERIMENTAL RESULTS}

The investigation was performed for 12 samples. Each sample had previously been decapsulated to provide a laser beam to SEL-sensitive areas.

The brief results are shown in Table 2. For samples $1 \ldots 8$, the maximum current in SEL state was limited to 1A. For samples 9...12, there were no current limits, supply current limit in SEL state was set by DUT and was up to $1.6 \mathrm{~A}$.

The results in Table 1 allow making the following preliminary suggestions:

1. Number of SEL, which is enough for the irreversible failure, depends on time in latch-up state in each SEL in series. Increasing the time in latch-up state results in decreasing "damage" number of SEL.

2. Amount of SEL, which is enough for the irreversible failure, depends on the maximum current in the latch-up state, in each SEL in series. Increasing the maximum current in the latch-up state results in decreasing "damage" number of SEL. 
3. For ADT7518 the irreversible failure (in this case "failure" means the ADC characteristic shift) was observed earlier than the irreversible supply current increase.

Table 2. Brief experimental results for ADT7518

\begin{tabular}{|c|c|c|c|c|c|}
\hline Sample \# & Stage & SEL amount & $\begin{array}{c}\text { Time } \\
\text { in latch-up state, } \mathrm{s}\end{array}$ & $\begin{array}{l}\text { Supply current increase } \\
\text { after SEL, mA }\end{array}$ & $\begin{array}{c}\text { Operation after SEL } \\
\text { (“+”-OK,"-“-failure ) }\end{array}$ \\
\hline 1 & 1 & 1 & 10 & 39 & - \\
\hline \multirow{3}{*}{2} & \multirow{3}{*}{1} & 1 & 5 & 0 & + \\
\hline & & 2 & 5 & 0 & - \\
\hline & & 6 & 5 & 26 & - \\
\hline \multirow{2}{*}{3} & \multirow{2}{*}{1} & 3 & 4 & $\mathrm{O}$ & + \\
\hline & & 4 & 4 & 24 & - \\
\hline \multirow{3}{*}{4} & \multirow{3}{*}{1} & 3 & 4 & $\mathrm{O}$ & + \\
\hline & & 4 & 4 & $\mathrm{O}$ & - \\
\hline & & 5 & 4 & 22 & - \\
\hline \multirow{2}{*}{5} & \multirow{2}{*}{1} & 51 & 2 & 0 & + \\
\hline & & 52 & 2 & 0 & - \\
\hline \multirow{3}{*}{6} & \multirow{3}{*}{1} & 2 & 4 & 0 & + \\
\hline & & 3 & 4 & 0 & - \\
\hline & & 17 & 4 & 34 & - \\
\hline \multirow{3}{*}{7} & \multirow{3}{*}{1} & 6 & 3 & 0 & + \\
\hline & & 7 & 3 & 24 & + \\
\hline & & 8 & 3 & 24 & - \\
\hline \multirow{3}{*}{8} & \multirow{3}{*}{1} & 44 & 2 & 0 & + \\
\hline & & 45 & 2 & 0.6 & + \\
\hline & & 52 & 2 & 26 & + \\
\hline \multirow{2}{*}{9} & \multirow{2}{*}{1} & 1565 & 1 & $\mathrm{O}$ & + \\
\hline & & 1566 & 1 & 0 & - \\
\hline \multirow{2}{*}{10} & \multirow{2}{*}{1} & 2 & 3 & $\mathrm{O}$ & + \\
\hline & & 3 & 3 & 550 & - \\
\hline \multirow{4}{*}{11} & \multirow{2}{*}{1} & 5 & 1.5 & 0 & + \\
\hline & & 6 & 1.5 & o & - \\
\hline & \multirow{2}{*}{2} & 1 & 4 & 0 & - \\
\hline & & 2 & 4 & 130 & - \\
\hline \multirow{6}{*}{12} & \multirow{4}{*}{1} & 2 & 2 & $\mathrm{O}$ & + \\
\hline & & 3 & 2 & $\mathrm{O}$ & - \\
\hline & & 44 & 2 & $\mathrm{O}$ & - \\
\hline & & 45 & 2 & 0.6 & - \\
\hline & \multirow{2}{*}{2} & 8 & 3 & 0.6 & \\
\hline & & 9 & 3 & 126 & - \\
\hline
\end{tabular}

up; moreover, probability of SEL series is proportional

\section{DISCUSSION}

The dependence of "damage" SEL number $N$ from time in latch-up state $t$ is in good correlation with Arrhenius law (1) (see Fig. 3):

$$
N=1+a \cdot e^{\frac{b}{t}},
$$

where $a$ - proportional coefficient, $b-$ so-called "activation energy" of processes which are accompanying the latch-up.

Such a result is expectable in the case of thermal basis of processes during latch-up.

Statistically, each SEL may be interpreted as an independent occurrence. Consequently, each following SEL increases the probability of failure (or damage) of IC. For a given time in latch-up state, the failure probability tends to 1 when number of SEL tends to the experimentally found "damage" amount. The reciprocal of the "damage" amount is the coarse estimation of the failure probability after single latch- to the number of SELs in series.

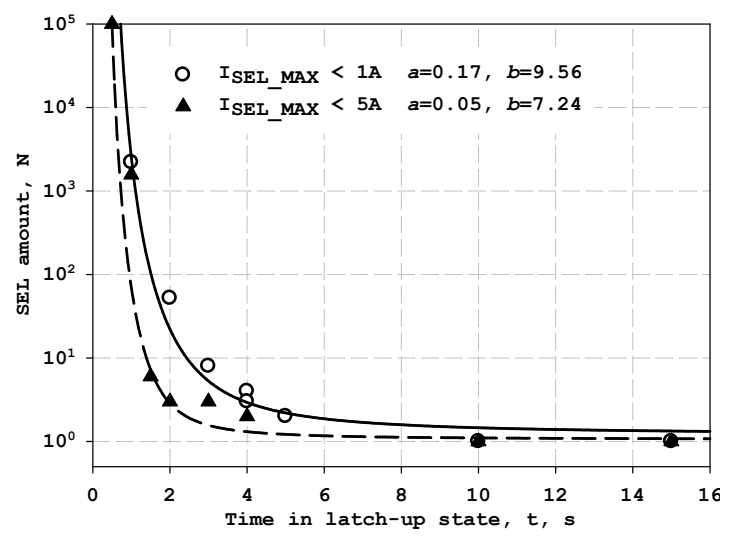

Figure 3. The dependence of "damage" SEL number $N$ from time in latch-up state $t$. Approximation is the Arrhenius law 
The dependence of reciprocal of the "damage" amount $n$ from time in latch-up state $t$ is in the good correlation with Weibull distribution (2) (see Fig. 4):

$$
n=1-e^{\left(\frac{t-a}{b}\right)^{c}},
$$

where $a$ - shift coefficient, $b$ - scale coefficient, $c-$ form coefficient.

The dependence is saturating on 1 - maximum failure probability after single latch-up. The threshold of dependence is the maximum time in latch-up state, for which any number of SEL does not result in failure or damage.

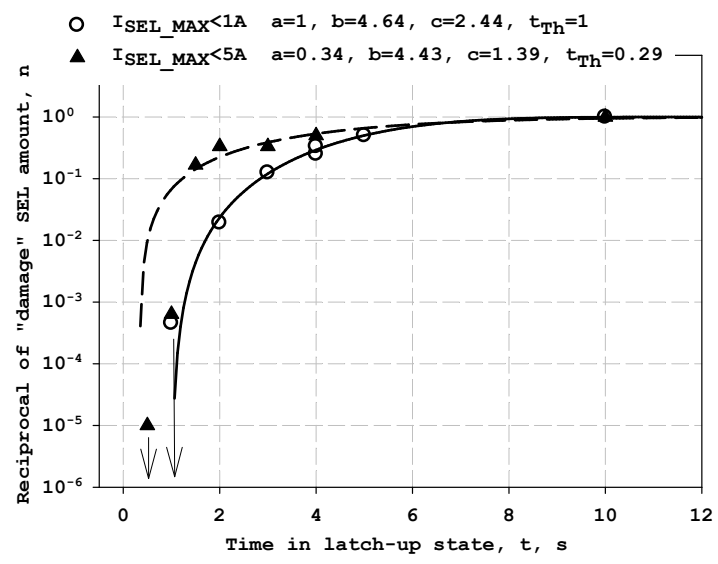

Figure 4. The dependence of reciprocal of "damage" SEL amount $n$ from time in latch-up state $t$. Approximation is the Weibull distribution

\section{CONCLUSION}

Thus, the approach to estimation of failure probability after series of SEL as the function of number of SEL and current in latch-up state is suggested. To make it, one needs to obtain the experimental dependence of reciprocal of SEL amount as a function of time in latch-up state. Using Weibull approximation of this dependency, one can estimate its threshold - the maximum "safe" time to parry the latch-up.

This approach may be used not only to SEL effect, but to all "cumulative" effects. In any case, however, one must perform preliminary studies to prove the effectiveness of supposed approach.
The next steps of this study are (a) to find "cumulative" SEL in another types of modern integrated circuits, and (b) to investigate effects of another nature with similar behavior.

\section{REFERENCES}

1. R. H. Maurer, M. E. Fraeman, M. N. Martin, D. R. Roth, "Harsh Environments: Space Radiation Environment, Effects, and Mitigation," Johns Hopkins APL Technical Digest, vol. 28, no. 1, 2008 .

Retrieved from:

http://www.jhuapl.edu/techdigest/TD/td2801/Maurer. $\mathrm{pdf}$ Retrieved on: Jan. 13, 2017

2. S. Duzellier, G. Hopkinson, J. C. Rodriguez "Radiation effects analysis (subdivided into total ionising dose, displacement, damage, and single-event effects)," presented at the RADECS-2003 short course, Noordwijk, The Netherlands, 2003.

3. K. LaBel, L. Cohn, "Radiation Testing and Evaluation Issues for Modern Integrated Circuits," in Proc. RADECS-2005, Cap d'Agde, France, 2005.

Retrieved from: https://radhome.gsfc.nasa.gov/radhome/papers/radec s05_sc.pdf Retrieved on: Feb. 3, 2017

4. H. N. Becker, T. F. Miyahira, A. H. Johnston, "Latent Damage From Single-Event Latchup," in Proc. Single Event Effects Symposium, Manhattan Beach, (CA), USA, 2002. Retrieved from: https://trs.jpl.nasa.gov/bitstream/handle/2014/8510/ 02-1001.pdf? sequence $=1$ \&isAllowed $=y$ Retrieved on: Feb. 5, 2017

5. S. H. Voldman, Latchup, 1st ed., Chichester, UK: J. Wiley and Sons, 2007. DOI: 10.1002/9780470516171

6. Hi-Rel Latchup Current Limiter, 3D plus, Buc, France, 2013.

Retrieved from: http://www.3dplus.com/data/doc/products/references/3dfp_0608 5 . pdf Retrieved on: Feb. 5, 2017

7. A. A. Pechenkin, "SEE radiation hardness assurance procedure of electronic components and systems," in RADECS-2015 short course, Moscow, Russia, 2015

8. PXI Advisor - National Instruments, National Instruments, Austin (TX), USA.

Retrieved from:

http://ohm.ni.com/advisors/pxi/pages/common/intro. xhtml

Retrieved on: Feb. 3, 2017 